\title{
A Culture of Safety: Read Beyond Academic Textbooks and Specialty Journals to Imbibe Best Practices of Other Professions
}

\author{
Nakul Uppal ${ }^{1}$ (i)
}

Received: 23 October 2018/ Accepted: 12 November 2018/Published online: 16 November 2018

(c) The Association of Oral and Maxillofacial Surgeons of India 2018

Maxillofacial surgery, like other surgical specialties, poses risks to patients in terms of known complications. Over decades, our specialty has striven hard to minimize morbidity and achieve excellence in outcomes. However, risks do remain; for example, facial nerve injury is a known risk in surgical access to the temporomandibular joints. Surgical risks aside, complications from carelessness constitute an entirely different subset of morbidity. As surgeons, we have a moral responsibility to our patients, our residents, and ourselves to minimize our avoidable errors, and to accomplish that, let us briefly ponder upon professions other than surgical practice and how they deal with risks and errors.

Humanity's responses to accidents vary. An airline crash is sensational news for days. Road accidents kill the equivalent daily of several passenger aircraft, but these are "quieter". Their daily occurrence lends mundaneness. Bad patches of road claim lives for years, with little correction. There is a cultural angle to how a society reacts to accidents. This indifferent reaction can transcend into health care.

At a Delhi hospital, two stillborn babies were parceled into plastic bags and-horrifyingly—one infant turned out to be alive. How were vital signs missed? More worryingly, were vital signs even checked? Newspapers report children falling into boiling sambhar on school premises. We hear of "selfie deaths" from falling off cliffs or overbalancing into rivers. Returning from a rural health camp, my intern colleague lost her life when she fell out of the

Nakul Uppal

drnakul@gmail.com

1 Department of Dentistry and Maxillofacial Surgery, All India Institute of Medical Sciences, Raipur, India doorway of the bus. Why cannot a bus door be kept closed? When my spouse asks me why I follow morbid news, I reply that I do so as a maxillofacial surgeon to learn how other professions minimize errors.

How is the above series of accidents applicable to better practice of health care? Students are expected to demonstrate mastery over curriculum by satisfactory performance in theoretical and practical examinations. However, beyond testing prescribed by councils, is something else required? To answer that, reflect upon this list of medical errors: "wrong tooth extracted"..... "healthy leg amputated"..... "NICU babies die after oxygen supply finishes"... ... "patient dies as trainee injects insulin into i.v.".. [1]. How should healthcare professionals react to errors? In India, we set up inquiry committees. Seldom are errors prevented from repeating. Generic instructions do little to improve safety. Guidelines to prevent errors are missing in Indian training just as formal safety has low visibility in hospitals, wards, OTs, ICUs, dental clinics, and laboratories [2]. There are centers of excellence which have brought down errors to negligible percentages, but these are niche, and not the trend. Many parts of India culturally have a laid-back attitude to discipline, hygiene, punctuality, and adherence to protocols. (A notable exception is Kerala where people and public spaces are neater.) When formal guidelines and strict rules are missing at hospitals, healthcare professionals carry personal habits to work. I remember a meticulous lady anesthesiologist whose fastidiousness and biting sarcasm had operating room personnel on their toes. A less fussy professional may carry slackness to the workplace, compromising on hand hygiene, legible writing, and punctuality in theater.

How do other professionals manage the daily spectre of errors? One sector with low error tolerance is aviation. Airline mishaps are rare. Mumbai airport handles over 
1000 flights a day: a world record. Consider the precise system, that every few minutes a large jet loaded with thousands of liters of inflammable fuel screams off a narrow strip of tarmac without colliding with other aircraft. How are rates of mishaps so low that most air incidents are near misses than crashes? The answer lies in the checklist. Atul Gawande, in The Checklist Manifesto, explains how airline pilots use checklists to minimize errors. The basic concept is of tediously verifying what a person is already innately familiar with. Near misses or deviations from flight path are investigated, and interrogation, review of data, derostering, and grounding of pilots is done. These rigid rules do not reduce job satisfaction. Rather, many pilots I talk to are cheerful professionals who love their work; protocols are a part of the job. The World Health Organization has devised checklists for hospitals, inspired by aviation. We also need a system of threatened punitive action if safety protocols are not followed. And to understand why, let us examine the military.

Tremendous, consistent discipline makes up India's military officer: a combat soldier-scholar. The schedule is grueling: drill square parade, daily runs, competitive physicals, plenty of administration, team sports, and a considerable amount of studies, courses, and examinations, all with fanatical adherence to time. What really sets the military apart is the provision in the Army Act for punitive action. An officer in uniform can be reprimanded, punished, chargesheeted, marched up, issued disciplinary action, etc., all for failure to comply with rules. Some brand this operant conditioning as a lack of human rights. In fact, the soldier wielding a weapon needs to have the highest level of competence in handling equipment, physical fitness to overcome fatigue, and mental strength to never misuse a weapon. Our private dental, medical, and nursing colleges charge such high fees that institutions pander to students' whims and brag of " $100 \%$ pass rates" at admission fairs. One of my favorite moments at a conference was at the stall of the Royal College examinations; they assured me that barely $30 \%$ passed! The military makes rigorous practice a mantra. Infantry soldiers strip and reassemble a rifle hundreds of times till the task is accomplished blindfolded. Soldiering, which we mistakenly believe involves more brawn than brain, transcends mental memory and enters the realm of muscle memory. An example of this phenomenon in health care is technicians who flawlessly draw blood in thousands of venipunctures. Imagine a curriculum where medical students are apprenticed briefly to experts to learn their art. Surgeons repeatedly performing procedures (the joint replacement specialist) develop a mastery of steps and achieve assembly-line-like efficiency in operative tasks. Malcolm Gladwell in his inspiring narrative Outliers explains that sports persons become award-winning athletes when they follow the 10,000-Hour
Rule. If Serena Williams-style obsessive practice of the same tasks makes for perfection, is it right to decry the death of general practice and criticize super-specialty residency? Rather, focused fellowships by the AOMSI are a progressive step. And is a reprimand to a resident wrong or a reflection of a weak system?

My third example is the automotive sector. The automobile industry regularly issues recalls for suspected flaws. Recalls, rather than shake consumer confidence, actually inspire a feeling of safety matters. But corporations in health care are in the news for lawsuits (e.g., litigation against J \& J for talcum powder's cancer link) or for defective implants ( $\mathrm{J} \& \mathrm{~J}$ 's ongoing advisory for recall of defective implants). On service visits to my car's workshop, I enjoy their systematic approach: There's a reminder call (think: the hospital reception makes a gentle phone call to a patient for review); uniformed and polite service managers update essential data (analogous to patient history!) on tablets; a checkup, under-bonnet assessment, and test-drive (physical examination!) is done and diagnostic information from scanning the OBD port (investigations and imaging!) are recorded. Cars are allocated to fully equipped service bays, akin to Procedure Rooms. By the end of service, consumables are replaced and the vehicle readied. We do not hear of car customers beating up workshop mechanics. If surgical practice could borrow from corporate-style car care, there might be fewer incidents of doctors assaulted by angry relatives. Imagine the zen-like tranquility that patients could also experience.

The fourth example is of the software industry. India's software writers' lack of genuine wildcard creativity (think Steve Wozniak or Linus Torvald) is made up by lower costs in writing code for clients. Software as a virtual product can be run through multiple layers of simulations before release for use in applications on which human lives matter (e.g., drive-by-wire technology behind modern cars' accelerator pedals). What if we could simulate every invasive procedure? Software is the key. For decades, commercial pilots have trained on multi-million-dollar simulators before flying real human passengers. A trainee pilot can practice an engine flameout, severe weather, instrument landing system failure, etc, all of this hundreds of times in a closed cockpit mock-up that pitches about on its hydraulic mounts simulating banking, turbulent flight, or a hard landing! With sophisticated personal computing (try out the free-to-use aircraft simulator at Chennai airport) and aggressive social media trolling, it is a matter of time before it becomes morally necessary for residents to practice on advanced simulation with haptic feedback before moving on to human patients. This exists in the West; India can innovate low-cost medical simulation.

Verbal communication occurs differently across professions. Pilots and air traffic control "copy" instructions. 
Repeating a phrase verbatim confirms that it has been comprehended correctly by the receiver. In the military, telecommunication equipment permits either talking or receiving, but not simultaneously. Alphabets are spoken aloud with phonetic names [3]. So, LCV would be read aloud as Lima-Charlie-Victor. Spoken this way, accents and different pronunciations cause fewer errors across radio static. Contrast this with our OTs: we are masked, muffled and looking away from each other, precluding lip reading. And so, wrong instruments are passed until the surgeon and scrub nurse understand subtle nuances of each other's' speech styles influenced by vernacular dialect accents.

Nations are categorized as "developing" or "developed" based on indices, typically Gross Domestic Product and Per Capita Income. A better index might instead welldesigned safety protocols. By that yardstick, India is a mixed bag, "developed" in many spheres and "developing" in public health, sanitation, safety, excellence, consistency, and ethical surgical practice. We lag behind developed economies in measuring healthcare outcomes. We shy away from voicing aloud our doubts and questioning our competencies. Measuring outcomes is something we grow up with through school tests and higher education examinations. Why then the anathema toward measuring outcomes? It could be a multitude of reasons: overburdened and unstructured work schedules of doctors in public hospitals, characteristics of PG training...or just our sociocultural reluctance to accept blame. A more cerebral effort in performing each step of our daily work practice might help. Steve Jobs, addressing Stanford students in 2005, invokes the "Stay Hungry. Stay Foolish" slogan of the 1974 edition of the Whole Earth Catalog, in other words, to assume no wisdom and constantly learn. The Japanese firm Toyota pioneered kaizen, meaning continuous improvement, helping the nation climb to spectacular industrial might after a humiliating defeat in World War II [4].

Our Prime Minister's call for a Swachh Bharat can be a start to raise surgical safety in India. Our specialty can rise further by embracing safety as a core value and an integral part of training. Unhygienic surroundings, untidiness, and tardiness in hospitals are incompatible with a push for zeroerror health care. Should we follow up on medical errors with PWD-type apathy or do we aim for military-like fastidiousness? Indian culture already has the ethos to attain excellence: The Japanese have their Souji; India has Janmabhoomi. Delicate bonsai and exquisite kanji are matched by madhubani and chikankari. What we need to do is bring perfectionist philosophy into mainstream consciousness so more people dwell more often on excellence.

Risks are common to all professions, whether aviation, manufacturing or construction. Risk analysis and risk management is a science that Indian health care needs to embrace. Accidents in oral and maxillofacial surgery (e.g., vasovagal syncope after a difficult third molar removal) can be predicted by paradigms such as the Swiss Cheese Model, which theorizes that many accidents can be anticipated if holes in the cheese (weaknesses in a system) are allowed to line up [5]. Errors then trickle past the four barriers of organizational influences (high patient volumes precluding good history), supervision (absence of experienced supervisors), preconditions (fatigued and fasting patient) and specific acts (inadequate local anesthesia). Fewer errors in surgical practice will happen when we surgeons take an interest in other professions and see how they minimize their errors. To imbibe best practices in Indian health care, let us start to look around and read beyond our textbooks and journals.

\section{Compliance with Ethical Standards}

Conflict of interest The author has no conflict of interest to declare.

\section{References}

1. https://www.indiatimes.com/news/india/5-recent-incidents-of-med ical-negligence-show-that-doctors-need-to-wake-up-from-slum ber-344053.html. Accessed 10 Oct 2018

2. Akhter S 5.2 million medical errors are happening in India annually. https://health.economictimes.indiatimes.com/news/indus try/5-2-million-medical-errors-are-happening-in-india-annuallydr-girdhar-j-gyani/53497049. Accessed 10 Oct 2018

3. https://en.wikipedia.org/wiki/NATO_phonetic_alphabet. Accessed 10 Oct 2018

4. Knechtges P, Decker MC (2014) Application of kaizen methodology to foster departmental engagement in quality improvement. J Am Coll Radiol 11:1126-1130

5. The Swiss cheese model of accidents (Bali R 2018, personal communication, 15 October) 